\title{
Quantitative multidimensional phenotypes improve genetic analysis of laterality traits
}

\author{
Judith Schmitz $\mathbb{D D}^{1}$, Mo Zheng ${ }^{2}$, Kelvin F. H. Lui $\mathbb{D}^{3}$, Catherine McBride ${ }^{2}$, Connie S.-H. Ho ${ }^{4}$ and Silvia Paracchini $\mathbb{D}^{1 凶}$ \\ (c) The Author(s) 2022
}

Handedness is the most commonly investigated lateralised phenotype and is usually measured as a binary left/right category. Its links with psychiatric and neurodevelopmental disorders prompted studies aimed at understanding the underlying genetics, while other measures and side preferences have been less studied. We investigated the heritability of hand, as well as foot, and eye preference by assessing parental effects ( $n \leq 5028$ family trios) and SNP-based heritability (SNP- ${ }^{2}, n \leq 5931$ children) in the Avon Longitudinal Study of Parents and Children (ALSPAC). An independent twin cohort from Hong Kong ( $n=358$ ) was used to replicate results from structural equation modelling (SEM). Parental left-side preference increased the chance of an individual to be left-sided for the same trait, with stronger maternal than paternal effects for footedness. By regressing out the effects of sex, age, and ancestry, we transformed laterality categories into quantitative measures. The SNP- $\mathrm{h}^{2}$ for quantitative handedness and footedness was 0.21 and 0.23 , respectively, which is higher than the SNP- $\mathrm{h}^{2}$ reported in larger genetic studies using binary handedness measures. The heritability of the quantitative measure of handedness increased (0.45) compared to a binary measure for writing hand $(0.27)$ in the Hong Kong twins. Genomic and behavioural SEM identified a shared genetic factor contributing to handedness, footedness, and eyedness, but no independent effects on individual phenotypes. Our analysis demonstrates how quantitative multidimensional laterality phenotypes are better suited to capture the underlying genetics than binary traits.

Translational Psychiatry (2022)12:68; https://doi.org/10.1038/s41398-022-01834-z

\section{INTRODUCTION}

The cerebral hemispheres differ in function and structure underpinning specialisation for cognition, perception, and motor control [1]. For instance, language is predominantly processed in the left hemisphere in most individuals [2] and the planum temporale typically shows a pronounced structural leftward asymmetry [3], although there is little evidence for a strong association between the two forms of asymmetry [4]. Neurodevelopmental disorders such as dyslexia [5, 6], schizophrenia [7], or autism spectrum disorder (ASD) [8] have been associated with a higher prevalence of atypical planum temporale asymmetry.

The most commonly studied lateralised trait is handedness. Worldwide, around $10 \%$ of the general population is left-handed with slight geographical variation [9], likely influenced by cultural factors $[10,11]$. Meta-analyses have confirmed higher rates of leftor non-right-handedness in ASD [12] and schizophrenia [13]. A genetic influence on handedness has been inferred from family and adoption studies [14]. For instance, the probability of lefthandedness increases with the number of left-handed parents [15]. Twin studies reported slightly higher rates of concordance in monozygotic (MZ) compared to dizygotic (DZ) twins $[16,17]$ and provided heritability estimates of around $0.25[18,19]$.

Family studies have suggested differential effects of fathers and mothers to their offspring's handedness. A stronger maternal than paternal effect was repeatedly found in biologically related parent-offspring trios $[20,21]$ and a similar trend was observable in an adoption study [22]. A maternal effect on non-righthandedness was also found in 592 families, where a paternal effect was only detectable in males [23].

A recent large-scale genome-wide association study (GWAS; $n \sim$ $2 \mathrm{M}$ ) estimated that up to $6 \%$ of the variance in left-handedness and up to $15 \%$ of the variance in ambidexterity are explained by common genetic markers [24]. As in most large-scale laterality studies, handedness was assessed as hand preference for writing, leading to three categories: right, left or both. The "both" category identifies individuals who say that they can write equally well with both hands, referred to as ambidextrous. However, a single task cannot identify mixed-handed individuals who prefer different hands for different activities. Instead, self-report questionnaires such as the Edinburgh Handedness Inventory (EHI) [25] assess the preferred hand for several manual activities and therefore capture both mixed-handed and ambidextrous individuals. A GWAS on brain imaging parameters $(n=32,256)$ revealed that genetic markers associated with structural brain asymmetries overlapped with markers previously associated with writing hand preference. Moreover, genetic factors involved in brain asymmetry overlap with neurodevelopmental and cognitive traits such as ASD, schizophrenia, educational attainment (EA) [26], and intelligence (IQ) [27]. These data suggest a general mechanism for the establishment of left/right asymmetry which is also important for

\footnotetext{
${ }^{1}$ School of Medicine, University of St Andrews, St Andrews, Scotland. ${ }^{2}$ Department of Psychology, The Chinese University of Hong Kong, Hong Kong, China. ${ }^{3}$ Wofoo Joseph Lee Consulting and Counselling Psychology Research Centre, Lingnan University, Hong Kong, China. ${ }^{4}$ Psychology Department, The University of Hong Kong, Hong Kong, China. email: sp58@st-andrews.ac.uk
} 
neurodevelopmental outcomes. Therefore, the analysis of other lateralised preferences will contribute to the understanding of such general mechanisms.

Foot and eye preference have received considerably less attention, even though associations with neurodevelopmental disorders have been reported as well. For example, we found an increased prevalence of non-right-footedness in neurodevelopmental and psychiatric disorders $\left(n_{\text {cases }}=2431, n_{\text {controls }}=116,938\right)$ [28]. Smaller studies point to higher rates of left eye preference in schizophrenia $\left(n_{\text {cases }}=88, n_{\text {controls }}=118\right.$ [29]; $n_{\text {cases }}=68, n_{\text {controls }}$ $=944$ [30]) and ASD ( $\left.n_{\text {cases }}=37 ; n_{\text {controls }}=20\right)$ [31]. Warren et al. [32] reported heritability estimates for foot and eye preference to be 0.12 and 0.13 , respectively. In Japanese twins, Suzuki and Ando [33] provided heritability estimates for foot preference ranging from 0.08 to 0.24 and having one left-footed parent increased the probability of being left-footed [34]. These studies support a genetic component for foot and eye preference although there is variability in heritability estimates, probably resulting from small sample sizes.

We performed the largest heritability study to date for multiple side preferences in the Avon Longitudinal Study of Parents and Children (ALSPAC) and a twin cohort from Hong Kong to investigate the heritability of laterality phenotypes, their associations with one another, and their links to neurodevelopmental and cognitive outcomes.

\section{MATERIALS AND METHODS \\ Cohorts}

ALSPAC: ALSPAC is a population-based longitudinal cohort. Pregnant women living in Avon, UK, with expected dates of delivery from 1st April 1991 to 31st December 1992 were invited to take part, resulting in 14,062 live births and 13,988 children who were alive at 1 year of age [35, 36]. Informed consent for the use of data collected via questionnaires and clinics was obtained from participants following the recommendations of the ALSPAC Ethics and Law Committee at the time. Ethical approval for the study was obtained from the ALSPAC Ethics and Law Committee and the Local Research Ethics Committees. Please note that the study website contains details of all the data that is available through a fully searchable data dictionary and variable search tool (http://www.bristol.ac.uk/alspac/ researchers/our-data/).

Hong Kong: Study participants were recruited from the Chinese-English Twin Study of Biliteracy, a longitudinal study of primary school twin children starting in 2014 [37]. Participating children were recruited from Hong Kong primary schools and had Cantonese as their native language. Language and cognitive ability tests have been conducted for over four waves with a one-year interval between assessments. Laterality data were collected during the second wave of assessment.

\section{Participants and phenotypes}

ALSPAC: Laterality phenotypes were assessed for children based on maternal reports and for parents as self-report. Hand preference was assessed using eleven items for parents and six items for children. Foot preference and eye preference were assessed using four and two items, respectively, for parents and children. All items were rated on a 3-point scale (coded as left $=1$, either $=2$, right $=3$, see Supplementary Table S1). Two summary items (one in a right-mixed-left [R-M-L] classification and one in a right-left classification [R-L]) were derived from recoded mean values across non-missing items for hand, foot, and eye preference (see supplementary methods and Figs. S1-S3 for details). Mean ages of mothers, fathers, and children were $32.54(S D=4.42), 34.42(S D=5.60)$ and $3.55(\mathrm{SD}=0.07)$ at the time of assessment, respectively.

Hong Kong: The overall sample comprised $n=366$ twin children (183 twin pairs) with a mean age of 8.67 years $(S D=1.23)$. This sample included $81 \mathrm{MZ}$ pairs ( 37 male pairs and 44 female pairs) and $102 \mathrm{DZ}$ pairs ( 21 male pairs, 19 female pairs, and 62 opposite-sex pairs). Twin zygosity of samesex twins was determined by genotyping small tandem repeat (STR) markers on chromosomes $13,18,21, \mathrm{X}$ and $\mathrm{Y}$ by Quantitative FluorescencePolymerase Chain Reaction (QF-PCR).

Hand, foot, and eye preference were assessed using a modification of the EHI [25]. The questionnaire was translated into Chinese and included six hand preference items, one foot preference item, and one eye preference item. All items were read to participants by a trained research assistant as described in detail previously [38]. Items were coded to a 3-point scale and a R-M-L summary item was created for hand preference (see Supplementary Methods and Supplementary Fig. S4 for details).

\section{Genotype quality control (QC)}

ALSPAC: Children's genotypes were generated on the Illumina HumanHap550-quad array at the Wellcome Trust Sanger Institute, Cambridge, UK and the Laboratory Corporation of America, Burlington, NC, US. Standard QC was performed as described elsewhere [39]. In total, 9115 children and 500,527 SNPs passed QC filtering.

Hong Kong: Genotyping was performed using Illumina Human Infinium OmniZhongHua-8 v1.3 Beadchip at the Prenatal Genetic Diagnosis Centre and the Pre-implantation Genetic Diagnosis laboratory in the Prince of Wales Hospital and The Chinese University of Hong Kong, Hong Kong SAR. Standard quality control measures were carried out. Genetic variants with missing rate $>10 \%$, minor allele frequency $(\mathrm{MAF})<0.01$ and with significant deviation from Hardy-Weinberg equilibrium $\left(p<1 \times 10^{-6}\right)$ were excluded. Individuals with genotyping rates $<90 \%$ and outlying heterozygosity rates were excluded. In total, 911,178 SNPs passed QC filtering. Among the $n=366$ twin children, genotype data were available for $n=$ 358 (81 MZ pairs and $98 \mathrm{DZ}$ pairs).

\section{Parental effects}

We included parent-child trios from ALSPAC with complete phenotypic data on the summary items for hand, foot or eye preference after excluding one of each twin pair $(n=113)$ and children with physical disabilities $(n=65)$ or sensory impairments $(n=50)$, resulting in a sample size (number of trios) of $n_{\text {hand }}=5028, n_{\text {foot }}=4960$ and $n_{\text {eye }}=4762$ (see Supplementary Table S1).

For hand, foot, and eye preference, we first performed two logistic regression analyses using both parents' sidedness as a predictor (coded as $0=$ two right-sided parents, $1=$ one mixed-sided parent, $2=$ one leftsided parent, $3=$ two mixed-sided parents, $4=$ one mixed- and one leftsided parent, $5=$ two left-sided parents). This analysis was performed for child sidedness (coded as right $=0$, left $=1$ ) using both the A) R-M-L classification (excluding mixed-sided children and their parents) and the B) $\mathrm{R}-\mathrm{L}$ classification.

Next, we differentiated maternal and paternal effects by using maternal sidedness, paternal sidedness (both coded as right $=0$, mixed $=1$, left $=$ 2), and offspring sex, as well as interaction terms between maternal and paternal sidedness with offspring sex as predictors. We used the wald.test() function to test for a difference between maternal and paternal effects using the R-M-L and the R-L classification.

As non-paternity could affect these analyses, we reran the logistic regression analyses including only confirmed biological parent-offspring trios as confirmed by genotype data. Genotypes were available for $n=$ 1719 fathers. We used the R package Sequoia [40], which assigns parents to offspring based on Mendelian errors. Sequoia uses birth year and sex to decrease the number of potential relationships between individuals and to correctly infer parents and offspring. As the exact birth year of children and parents in ALSPAC was unknown to us, children's birth year was set to 1992 and parents' birth year was roughly estimated from the age of the assessment of laterality data. We selected 500 SNPs randomly from a subset that had MAF $>0.45$, high genotyping rate (missingness $<0.01$ ) and low linkage disequilibrium (LD; $\mathrm{r}^{2}<0.01$ within a $50 \mathrm{~kb}$ window). The 500 SNPs were spread across chromosomes 1-22. Sequoia confirmed paternity for $n=1624$ fathers. Among this subsample of 1624 trios, complete phenotypic data were available for 1161 trios for handedness, 1150 trios for footedness, and 1105 trios for eyedness (see Supplementary Table S1).

To assess the reliability of maternal reports, we performed a Spearman rank correlation analysis between hand preference for drawing (left/right) assessed by maternal report at age 3.5 and self-reported hand preference for writing at age $7.5\left(M_{\text {age }}=7.50\right.$ years; $\left.n=3129\right)$.

\section{Phenotypic analysis}

Unrelated children (genetic relationship $<0.05, n=5956$ ) with genomewide genetic and phenotypic data were selected for Genome-wide Complex Trait Analysis (GCTA) [41]. The same sample was used for phenotypic analysis. Sample sizes varied from $n=4630$ (foot used to pick up a pebble) to $n=5931$ (summary item for hand preference). 
Summary items in the R-M-L classification for hand, foot, and eye preference and 12 single items were residualised for sex, age, and the two most significant principal components:

$Y_{i}=\beta_{0}+\beta_{1} X_{1 i}+\beta_{2} X_{2 i}+\beta_{3} X_{3 i}+\beta_{4} X_{4 i}+\varepsilon_{i}$

Where $Y_{i}$ is the laterality summary item (coded as right $=0$, mixed $=1$, left $=2), \beta_{0}$ is the intercept, $\beta_{1}$ is the regression weight for offspring sex, $X_{1 i}$ is offspring sex, $\beta_{2}$ is the regression weight for offspring age in weeks, $X_{2}$ is offspring age in weeks, $\beta_{3}$ is the regression weight for $P C 1, X_{3 i}$ is $P C 1, \beta_{4}$ is the regression weight for $P C 2, X_{4 i}$ is $P C 2$, and $\varepsilon_{i}$ reflects random error.

Phenotypes were then inverse rank-transformed to achieve normally distributed phenotypes. Principal components were calculated based on directly genotyped $(\mathrm{MAF}<0.05)$ and $\mathrm{LD}$ pruned $\left(r^{2}<0.01\right.$ within a $50 \mathrm{~kb}$ window) SNPs (excluding high LD regions) using Plink v2. The rationale for including PCs in the phenotype transformation was based on the Geneticrelationship-matrix structural equation modelling (GRM-SEM) method which has been developed using the ALSPAC cohort [42]. As there is little population stratification in ALSPAC, the PC effect on the phenotypes is very small. Instead, higher scores indicated being left-sided, being female [43, 44], and younger age. Phenotypic correlations between rank-transformed items were calculated with Pearson correlation, applying FDR correction for 105 comparisons using the Benjamini-Hochberg method [45].

\section{Heritability estimates}

SNP- $\mathrm{h}^{2}$ was calculated for the transformed R-M-L summary items ( 3 ) and single items (12) using restricted maximum-likelihood (REML) analysis in GCTA [46], which compares phenotypic similarity and genotypic similarity based on a genetic-relationship matrix (GRM) in unrelated individuals. A GRM was estimated based on directly genotyped SNPs for unrelated children (genetic relationship $<0.05, n=5956$ ) using GCTA.

As a comparison, SNP-h ${ }^{2}$ was calculated for the untransformed categorical items using sex, age, and the first two principal components as covariates. We estimated SNP-h ${ }^{2}$ separately for left-sidedness (left vs. right, excluding mixed-sided individuals) and mixed-sidedness (mixed vs. right, excluding left-sided individuals).

Next, we estimated heritability from parent-offspring data [47]. Among the subsample with genomic data and confirmed paternity, we selected those with information on age at the time of laterality assessment, resulting in a sample of 1000 trios for handedness, 991 trios for footedness, and 957 trios for eyedness. Summary items in the R-M-L classification for hand, foot, and eye preference (coded as right $=0$, mixed $=1$, left $=2$ ) were transformed following the same procedure described above for the ALSPAC children. We estimated heritability by performing linear regression analyses using mean parental laterality as predictor and child laterality as the outcome:

$Y_{i}=\beta_{0}+\beta_{1} X_{i}+\varepsilon_{i}$

Where $Y_{i}$ is the transformed offspring laterality item, $\beta_{0}$ is the intercept, $\beta_{1}$ is the regression weight (heritability index), $X_{i}$ is the mean parental laterality, and $\varepsilon_{\mathrm{i}}$ reflects random error.

\section{SEM}

We applied GRM-SEM [42] to quantify shared and unique genetic factors among R-M-L summary items for hand, foot, and eye preference. This method has recently been used to study genetic associations among language and literacy skills in the ALSPAC cohort [48]. Equivalent to heritability analysis in twin research, GRM-SEM partitions phenotypic variance/covariance into genetic and residual components, but estimates genetic variance/covariance based on genome-wide genetic markers. We used the same GRM described above (based on directly genotyped SNPs for $n=5956$ unrelated children using GCTA). A GRM-SEM was fitted using the grmsem library in $R$ (version 1.1.0) using all children with phenotypic data for at least one phenotype. Multivariate trait variances were modelled using a saturated model (Cholesky decomposition). GRM-SEM was also used to estimate bivariate heritability, i.e. the contribution of genetic factors to the phenotypic covariance.

The heritability of laterality phenotypes was additionally estimated using a classical twin design that compares the similarity of $M Z$ to that of $D Z$ twins. Since $M Z$ twins share nearly all their genetic variants, whereas DZ twins share on average $50 \%$ of their genetic variants, any excess similarity of $M Z$ twins over DZ twins is the result of genetic influences. This method partitions phenotypic variance into that due to additive genetic $(A)$, shared environmental (C) and non-shared environmental influence (E). The variance attributed to each component can be estimated using the structural equation modelling (SEM) technique and the proportion of variance explained by the genetic influence $(A)$ is termed heritability. Phenotypes were transformed following the same procedure described for ALSPAC above. We fit a multivariate ACE model to the transformed phenotypes (handedness, footedness, and eyedness) and compared ACE with its constrained models, such as the AE model. Analyses were performed using the OpenMx software package 2.18.1 [49]. The script was adapted from the International Workshop on Statistical Genetic Methods for Human Complex Traits [50].

\section{Polygenic risk score (PRS) analysis}

We conducted PRS analyses using summary statistics for handedness assessed as a binary trait, psychiatric and neurodevelopmental conditions (ASD, ADHD, bipolar disorder (BIP), schizophrenia (SCZ)), and cognitive measures (EA and IQ) using PRSice 2.3.3 [51]. PRS analyses were performed for hand and foot preference (which showed significant SNP- $\mathrm{h}^{2}$ ) in the ALSPAC cohort. The summary statistics for hand preference (left vs. right) were calculated after excluding individuals from 23andMe as well as ALSPAC from the original GWAS [24] sample. Summary statistics for ADHD [52], ASD [53], BIP [54], and SCZ [55] were accessed from the Psychiatric Genomics Consortium (PGC) website (https://www.med.unc.edu/pgc/dataindex/). Summary statistics for IQ [56] and EA [57] were accessed from the Complex Trait Genetics (CTG) lab website (https://ctg.cncr.nl/software/ summary_statistics), and the Social Science Genetic Association Consortium (https://www.thessgac.org/data), respectively.

PRS were derived from LD-clumped SNPs $\left(r^{2}<0.1\right.$ within a $250 \mathrm{~kb}$ window) as the weighted sum of risk alleles according to the training GWAS summary statistics. No covariates were included as phenotypes had been corrected for effects of age, sex, and ancestry. Results are presented for the best training GWAS $p$ value threshold (explaining maximum phenotypic variance) as well as GWAS $p$ value thresholds of $0.001,0.05,0.1$, $0.2,0.3,0.4,0.5$, and 1 . Results were FDR-corrected for 126 comparisons (7 training GWAS; 2 target phenotypes; $9 p$ value thresholds) using the Benjamini-Hochberg method [45].

\section{RESULTS}

\section{Parental effects}

We tested parental effects by assessing the percentages of non-rightsided (R-M-L) and left-sided (R-L) offspring as a function of parental sidedness in the whole sample and in trios with confirmed biological paternity. As expected, the percentage of non-right-sidedness and left-sidedness were highest in individuals with two non-right-sided or two left-sided parents, respectively (Supplementary Tables S2 [R-M-L] and S3 [R-L]). The percentage of non-right-sidedness and leftsidedness were higher in individuals with a non-right-sided or leftsided mother and a right-sided father than vice versa for all three traits. This effect was visible in both the whole sample (e.g. $31.23 \%$ vs. $25.83 \%$ for non-right-handedness, see Supplementary Table S2) and in the subset with confirmed biological paternity (e.g. $33.33 \%$ vs. 25.37\%, see Supplementary Table S2).

Second, we ran logistic regression analyses in $n \leq 5028$ ALSPAC family trios. In the R-M-L classification $\left(n_{\text {hand }}=4248, n_{\text {foot }}=3242\right.$ and $n_{\text {eye }}=3050$ ), parental sidedness predicted hand, $X^{2}(5)=39.5$, $p=1.9 \times 10^{-7}$, foot, $X^{2}(5)=59.9, p=1.3 \times 10^{-11}$, and eye preference, $X^{2}(5)=27.4, p=4.8 \times 10^{-5}$. In the R-L classification ( $n_{\text {hand }}$ $=5028, n_{\text {foot }}=4960$ and $n_{\text {eye }}=4762$ ), parental sidedness also predicted hand, $\mathrm{X}^{2}(2)=42.6, p=5.5 \times 10^{-10}$, foot, $\mathrm{X}^{2}(2)=69.1, p$ $=1.0 \times 10^{-15}$, and eye preference, $X^{2}(2)=14.6, p=6.9 \times 10^{-4}$. ORs show that having one or two left-sided parents increased one's chances to be left-sided for hand, foot, and eye preference in the R-M-L classification (Fig. 1A) and in the R-L classification (Fig. 1B). Analysis in the subsample with confirmed paternity $(n \leq 1161$ family trios) showed similar, although attenuated, parental effects for hand (R-M-L: $X^{2}(4)=14.9, p=0.005 ; R-L: X^{2}(2)=12.1, p=$ 0.002 ) and foot (R-M-L: $\mathrm{X}^{2}(4)=22.5, p=0.0002 ; \mathrm{R}-\mathrm{L}: \mathrm{X}^{2}(2)=19.1, p$ $\left.=7.1 \times 10^{-5}\right)$, but not for eye $\left(\mathrm{R}-\mathrm{M}-\mathrm{L}: \mathrm{X}^{2}(5)=5.3, p=0.380\right.$; R-L: $\left.\mathrm{X}^{2}(2)=2.7, p=0.250\right)$ preference (Supplementary Fig. S5). The full regression model outputs for the whole sample and for trios with confirmed paternity can be found in Supplementary Tables S4-S7. 


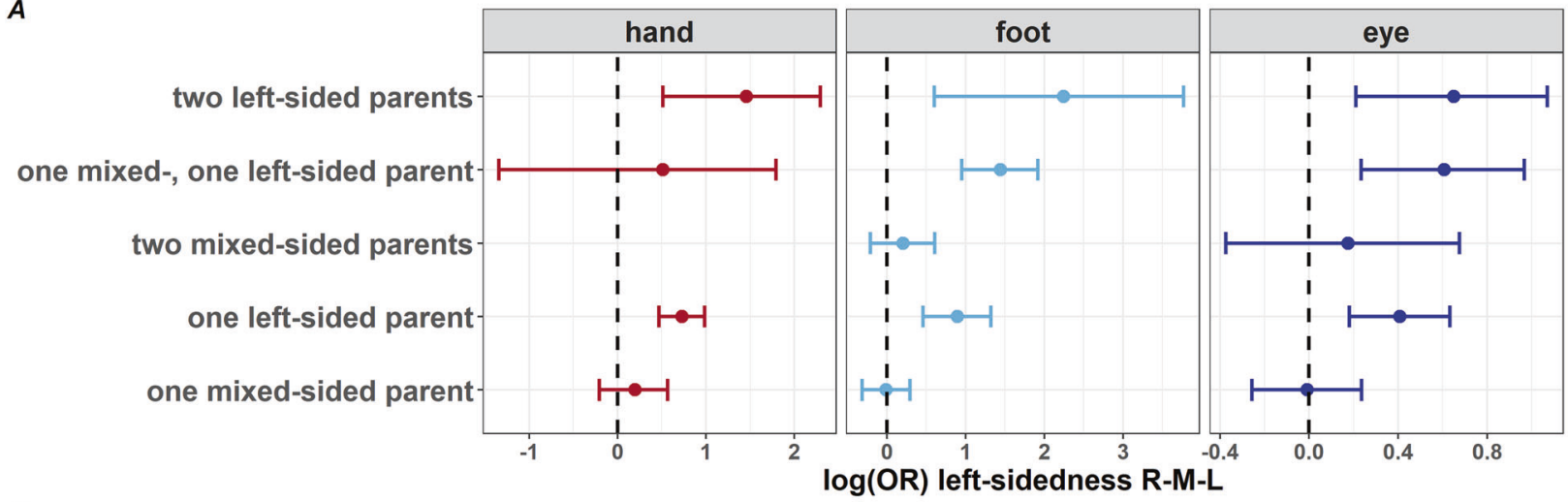

B

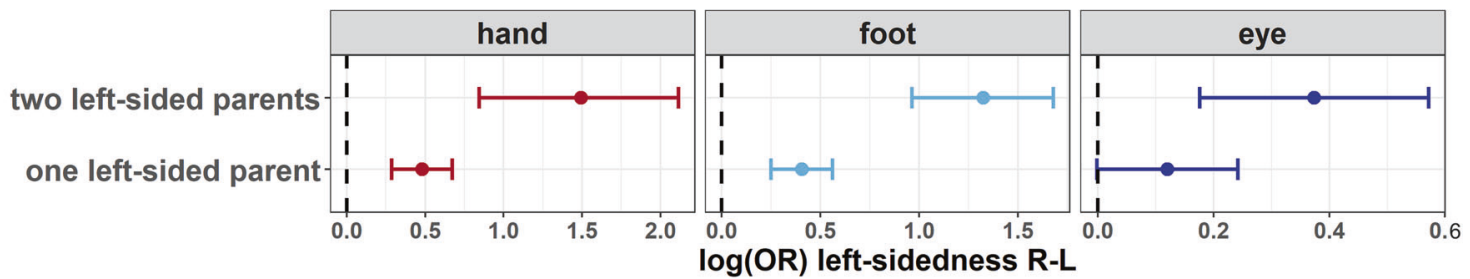

Fig. 1 Parental effects on child sidedness. ORs $[95 \% \mathrm{Cl}]$, resulting from logistic regression analysis.

Third, we investigated maternal and paternal effects and possible interactions with offspring sex. In the whole sample, Wald tests revealed a significant maternal effect on hand (R-M-L: $\left.\mathrm{X}^{2}(4)=38.9, p=7.4 \times 10^{-8} ; \mathrm{R}-\mathrm{L}: \mathrm{X}^{2}(2)=31.7, p=1.3 \times 10^{-7}\right)$, foot $\left(\mathrm{R}-\mathrm{M}-\mathrm{L}: \mathrm{X}^{2}(4)=52.7, p=9.8 \times 10^{-11} ; \mathrm{R}-\mathrm{L}: \mathrm{X}^{2}(2)=96.6, p<2.2 \times\right.$ $\left.10^{-16}\right)$, and eye preference (R-M-L: $\mathrm{X}^{2}(4)=38.3, p=9.7 \times 10^{-8} ; \mathrm{R}-\mathrm{L}$ : $\left.\mathrm{X}^{2}(2)=34.1, p=3.9 \times 10^{-8}\right)$. Paternal sidedness predicted hand $(\mathrm{R}-$ M-L: $\left.X^{2}(4)=10.3, p=0.036 ; \mathrm{R}-\mathrm{L}: \mathrm{X}^{2}(2)=12.3, p=0.002\right)$ and foot $\left(\mathrm{R}-\mathrm{M}-\mathrm{L}: \mathrm{X}^{2}(4)=15.1, p=0.005 ; \mathrm{R}-\mathrm{L}: \mathrm{X}^{2}(2)=6.0, p=0.049\right)$, but not eye preference (R-M-L: $\mathrm{X}^{2}(4)=4.6, p=0.330 ; \mathrm{R}-\mathrm{L}: \mathrm{X}^{2}(2)=0.6, p=$ $0.760)$. Wald tests contrasting maternal and paternal effects revealed a stronger maternal than paternal effect only for foot preference (R-M-L: $\mathrm{X}^{2}(1)=4.6, p=0.033$; R-L: $\mathrm{X}^{2}(1)=23.9, p=$ $\left.1.0 \times 10^{-6}\right)$. This effect was confirmed in the subsample with confirmed paternity (R-M-L: $\mathrm{X}^{2}(1)=8.4, p=0.004$; R-L: $\mathrm{X}^{2}(1)=10.0$, $p=0.002$ ). Although attenuated in the smaller subsample with confirmed paternity, this finding suggests a genuinely stronger maternal than paternal effect on footedness. In the whole sample, interaction terms between maternal/paternal sidedness and offspring sex revealed that in the R-L classification, maternal leftsidedness had a greater effect on left-footedness in girls compared to boys ( $\beta=0.49, \mathrm{SE}=0.19, z=2.55, p=0.011$ ), which was confirmed in the smaller subsample $(\beta=0.96, \mathrm{SE}=0.44, z=$ $2.17, p=0.030$ ). The full regression model outputs for both the whole sample and the subsample with confirmed paternity can be found in Supplementary Tables S8-S11.

Besides non-paternity, the reliability of the maternal report on laterality phenotypes could have affected our analysis. Correlation analysis showed a strong association between hand preference for drawing collected at 3.5 years of age and the self-reported hand preferred for writing at age $7.5(r=0.95,95 \% \mathrm{Cl}=[0.93,0.97], p<$ $\left.2.2 \times 10^{-16}\right)$. Among the 2838 children with a right-hand preference at age 3.5, seven reported a left-hand preference for writing at age 7.5. Of the 291 children with left-hand preference at age 3.5, 19 showed a right-hand preference for writing at age 7.5. Overall, $99.2 \%$ of individuals showed stable hand preference (see Supplementary Table S12), demonstrating the reliability of the maternal report.

\section{Transformed phenotypes}

Phenotypic correlation and genomic analyses (SNP- $h^{2}$ estimates, GRM-SEM and PRS analysis) were performed in unrelated children from the ALSPAC cohort $(n \leq 5931)$. Multivariate behavioural SEM analysis was performed in the Hong Kong twin sample $(n \leq 358)$. The absolute numbers and percentages of children with left, mixed, and right side preference for the three summary items in both cohorts are shown in Table 1.

By regressing out the effects of sex, age, and ancestry, we transformed laterality categories into quantitative measures using formula (1). We assessed phenotypic correlations for the transformed items in ALSPAC and the Hong Kong cohort. In ALSPAC, the single item that best captured the summary item was "hand used to draw" for hand preference $\left(r=0.87, t_{(5920)}=139.01\right.$, $\left.p<2.2 \times 10^{-16}\right)$, "foot used to stamp" for foot preference $(r=0.78$, $\left.t_{(5765)}=95.78, p<2.2 \times 10^{-16}\right)$, and "eye used to look through a bottle" for eye preference $\left(r=0.96, t_{(5469)}=249.61, p<2.2 \times\right.$ $\left.10^{-16}\right)$. In both cohorts, summary items showed positive correlations with each other (Supplementary Figs. S6 and S7). These correlations support a general left/right directionality captured by the different items.

\section{Heritability estimates}

We then tested the heritability of the transformed phenotypes. SNP- $\mathrm{h}^{2}$ of transformed laterality items ranged from 0.00 ( $p=$ $0.500)$ for "eye used to look through a bottle" to $0.42(p=8 \times$ $10^{-13}$ ) for "hand used to cut" (Fig. 2, Supplementary Table S13). The highest heritability estimate for summary measures was observed for footedness (SNP- $\mathrm{h}^{2}=0.23 ; p=2 \times 10^{-5}$ ), followed by handedness (SNP- $\left.h^{2}=0.21 ; p=1 \times 10^{-4}\right)$. There was no significant SNP-h ${ }^{2}$ for eyedness (SNP- $\mathrm{h}^{2}=0.00 ; p=0.469$ ).

For comparison, we estimated the SNP- $\mathrm{h}^{2}$ for the untransformed categorical items for left- and mixed-side preference categories. SNP- $\mathrm{h}^{2}$ for left-side preference ranged from $0.00(p=$ $0.500)$ for "foot used to climb a step" to $0.13(p=0.031)$ for "hand used to cut" (Supplementary Fig. S8A, Supplementary Table S14). SNP- $\mathrm{h}^{2}$ for mixed-side preference ranged from $0.00(p=0.500)$ for the hand preference summary item to $0.12(p=0.031)$ for "hand used to draw" (Supplementary Fig. S8B, Supplementary Table S15).

Parent-offspring regression run on the transformed summary items suggested heritability estimates of 0.27 for handedness $(95 \% \mathrm{Cl}=$ $\left.[0.11,0.42], p=5.6 \times 10^{-4}\right), 0.09$ for footedness $(95 \% \mathrm{Cl}=[0.01,0.17]$, $p=0.030)$, and 0.08 for eyedness $(95 \% \mathrm{Cl}=[-0.04,0.20], p=0.198)$.

Univariate SEM analysis in the Hong Kong cohort resulted in heritability estimates of 0.45 for handedness $(95 \% \mathrm{Cl}=[0.29$, 
Table 1. Children with left, mixed, and right side preference for each phenotype in ALSPAC (unrelated children) and the Hong Kong cohort (twin children).

\begin{tabular}{|lllllllll} 
& \multicolumn{1}{c}{ ALSPAC } & \multicolumn{2}{c}{ Hong Kong } & \\
& $\boldsymbol{n}$ & Left & Mixed & Right & $\boldsymbol{n}$ & Left & Mixed & Right \\
\hline Hand preference & 5931 & $471(7.9 \%)$ & $893(15.1 \%)$ & $4567(77.0 \%)$ & 358 & $20(5.6 \%)$ & $37(10.3 \%)$ & $301(84.1 \%)$ \\
\hline Foot preference & 5860 & $344(5.9 \%)$ & $2070(35.3 \%)$ & $3446(58.8 \%)$ & 358 & $31(8.7 \%)$ & $106(29.6 \%)$ & $221(61.7 \%)$ \\
\hline Eye preference & 5650 & $730(12.9 \%)$ & $2012(35.6 \% \%)$ & $2908(51.5 \%)$ & 357 & $95(26.5 \%)$ & $107(29.9 \%)$ & $155(43.4 \%)$
\end{tabular}

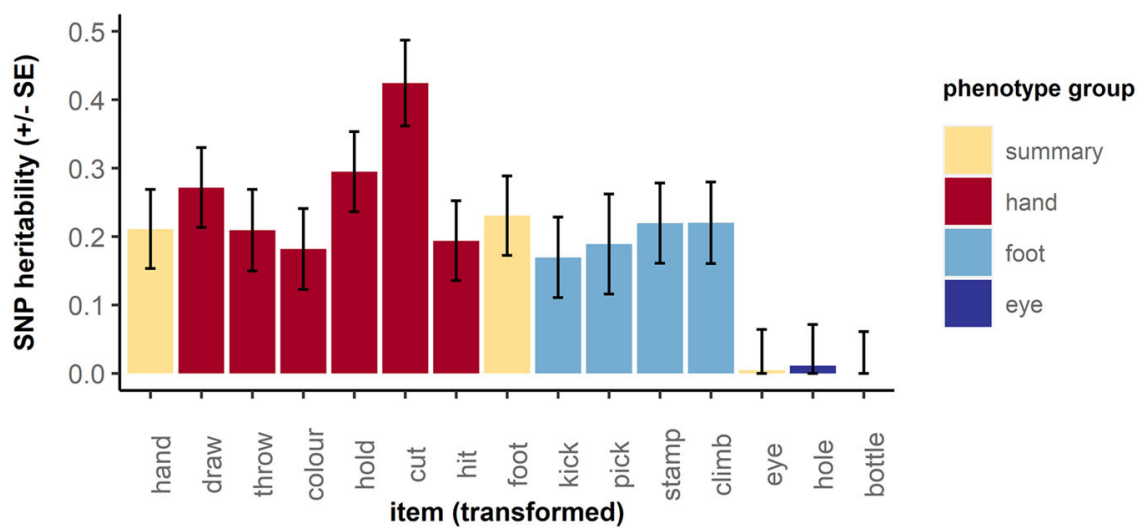

Fig. 2 SNP- $\mathbf{h}^{2}$ estimates for laterality measures after transformation into quantitative scores in ALSPAC. Results are shown for individual items and summary measures (yellow). Bars represent standard errors.
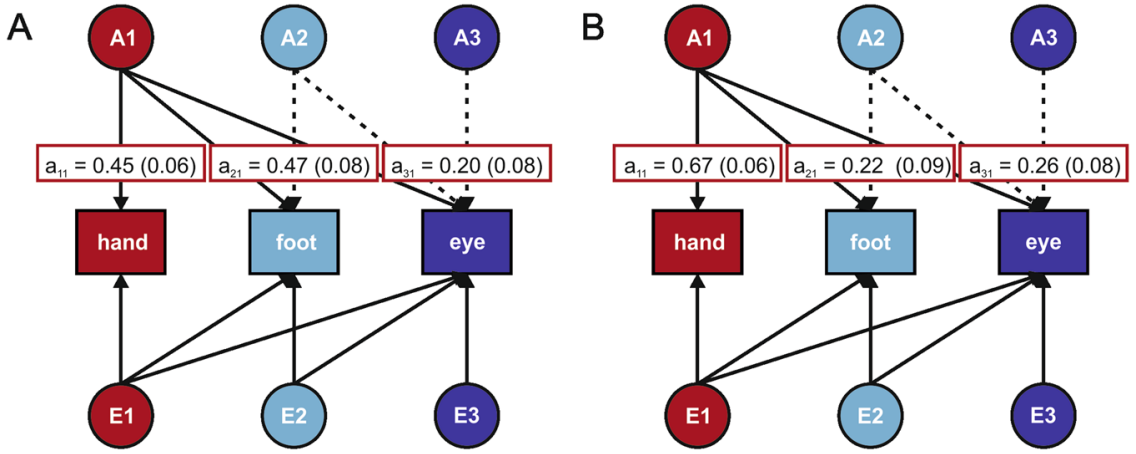

Fig. 3 Results of SEM analyses between laterality phenotypes. A Results of GRM-SEM in ALSPAC. B Results of behavioural SEM in the Hong Kong cohort. Circles on top and bottom indicate genetic (A) and environmental (E) factors, respectively. Coloured boxes indicate the phenotypes. Solid lines indicate significant path coefficients, dotted lines indicate non-significant path coefficients. White boxes indicate path coefficients and standard errors (SE) for significant genetic factors. The contour of the white boxes indicates the genetic factor (A1 in all cases).

$0.63]), 0.08$ for footedness $(95 \% \mathrm{Cl}=[0.00,0.25])$, and 0.08 for eyedness $(95 \% \mathrm{Cl}=[0.00,0.26])$. Therefore, the heritability estimates for the quantitative phenotypes were consistently higher than for categorical measures, both for SNP- ${ }^{2}$, parentoffspring, and twin SEM estimates.

\section{SEM}

Multivariate GRM-SEM analysis was performed on the transformed R-M-L summary items for handedness, footedness, and eyedness in ALSPAC (Fig. 3A). The squared path coefficient of genetic factor A1 explains genetic variance in hand preference $\left(a_{11}\right)$ and genetic variance that is shared with foot $\left(a_{21}\right)$ and eye preference $\left(a_{31}\right)$. A single genetic factor (A1) explained $20.36 \%$ of the phenotypic variance in handedness $\left(\mathrm{a}_{11}=0.45, p=2.4 \times 10^{-12}\right), 22.12 \%$ of the variance in footedness $\left(a_{21}=0.47, p=9.2 \times 10^{-10}\right)$ and $3.84 \%$ of the variance in eyedness $\left(a_{31}=0.20, p=9.2 \times 10^{-3}\right)$. All other path coefficients were non-significant, suggesting that one shared genetic factor (A1) contributes to a general left/right directionality across all three phenotypes.
Bivariate heritability analysis confirmed that shared genetic influences accounted for $36.7 \%$ of the phenotypic correlation between handedness and footedness $\left(p=6.6 \times 10^{-6}\right), 24.9 \%$ of the correlation of between footedness and eyedness $(p=0.020)$, and $26.2 \%$ of the correlation between handedness and eyedness $(p=0.020)$. We replicated these findings with multivariate behavioural SEM in an independent cohort $(n=358)$. In the Hong Kong cohort, $\mathrm{A} 1$ explained $44.30 \%(95 \% \mathrm{Cl}=[28.50,62.30])$ of the phenotypic variance in handedness $\left(\mathrm{a}_{11}=0.67, p<0.001\right), 5.00 \%$ $(95 \% \mathrm{Cl}=[0.20,15.30])$ of the variance in footedness $\left(\mathrm{a}_{21}=0.22, p\right.$ $=0.014)$, and $7.00 \%(95 \% \mathrm{Cl}=[0.80,18.20])$ of the variance in eyedness $\left(\mathrm{a}_{31}=0.26, p=0.003\right)$ (Fig. 3B). All other path coefficients were non-significant, consistent with results for ALSPAC.

\section{PRS analysis}

None of the PRS associations survived correction for multiple comparisons. The strongest association was found for PRS for IQ, suggesting that genetic predisposition towards higher IQ is associated with a tendency towards right-handedness 
$\left(\beta=-1159.21, \quad \mathrm{SE}=414.71, \quad \mathrm{PRS} R^{2}=0.13 \%, \quad p=0.005\right)$. PRS results for all $p$ value thresholds are reported in Supplementary Table S16.

\section{DISCUSSION}

We investigated the heritability of hand, foot, and eye preference using multiple approaches. To the best of our knowledge, this is the largest study conducted to date for multiple laterality measures in the same individuals. Our analysis of family trios showed that the probability of being left-sided increased for any left-sided parent on the same trait, with stronger effects for hand and foot, rather than eye preference, in line with previous reports $[15,34]$. Stronger maternal than paternal effects have been reported in studies focussing mainly on handedness [20, 23]. In ALSPAC, we found a stronger maternal than paternal effect for foot, but not hand or eye preference. This stronger maternal effect was detected in the whole sample ( $n=4960$ trios) and confirmed in the subset with genetically confirmed paternity ( $n=1150$ trios). Maternal/paternal effects could be explained with sex-linked genetic or parent-of-origin effects. For example, the imprinted LRRTM1 gene was found to be associated with handedness under a parent of origin effect [58]. Parent of origin effects might be more wide-spread than appreciated, but their detection requires family samples as opposed to the most commonly used singleton cohorts [59]. Few examples of parent-of-origin effects have been reported, for example for language-related measures [60-62]. Besides non-paternity, the reliability of the maternal report on laterality phenotypes could have affected our analysis. We confirmed strong correlation $(r=0.95)$ between the preferred hand for drawing assessed using maternal report at age 3.5 and self-reported preferred hand for writing in later childhood. The fact that more children switch hand preference from left to right [63] could indirectly suggest that switching attempts by parents or teachers have occurred at least until the mid 1990s. Overall, our analysis supports a genetic component underlying these laterality traits and highlights a specific maternal effect for footedness. The maternal effects could result from a higher genetic load required to manifest left-side preference in females. A similar buffering effect has been proposed to explain the higher prevalence of neurodevelopmental disorders in males [64].

Using transformed quantitative phenotypes [48], we estimated SNP- $\mathrm{h}^{2}$ for handedness, footedness, and eyedness to be $0.21,0.23$, and 0.00 , respectively. The heritability estimate for handedness is similar to what has been reported in behavioural twin studies $\left(\mathrm{h}^{2}\right.$ $=0.25)[18,19]$ but higher than observed in GWAS $\left(S N P-h^{2}=0.06\right)$ $[24,65,66]$ for categorical handedness. Instead, estimates for categorical phenotypes were non-significant, suggesting that the transformed phenotypes are better suited to detect the genetic component underlying lateralised traits than binary phenotypes. Accordingly, behavioural analysis in the Hong Kong twin cohort revealed a heritability estimate of 0.45 for the quantitative handedness phenotype - much higher than what has been observed for a categorical measure of writing hand (0.27) in the same cohort [38]. Parent-offspring regression in ALSPAC also showed significant heritability for handedness and footedness when using the quantitative phenotypes. We conclude that the quantitative phenotypes are better suited to capture the polygenic nature of handedness as expected under a liability threshold model [67]. The lack of association between the PRS derived from a recent large-scale GWAS for categorical handedness [24] suggests the influence of separate genetic factors. Lack of heritability for eyedness could reflect the poor quality of phenotype assessment, i.e. eyedness might be more difficult to assess and report accurately. Another possibility is that human eye preference does not have particular functional advantages and therefore the preferred side is less influenced by evolutionary forces and genetic factors. This is in contrast to other vertebrates such as bird [68] or fish species [69], where eye preference is involved in predator detection or social interaction.

Heritability estimates differed substantially between items used to assess handedness, footedness, and eyedness. We found the highest SNP-h2 for "hand used to cut" (with a knife). Previously, this item showed the weakest phenotypic correlation with the other questionnaire items $[70,71]$ and the highest heritability [33]. It has been proposed that summary items have reduced value to determine genetic factors involved in laterality [32]. This was true for the handedness measure, but conversely, we observed higher SNP- $\mathrm{h}^{2}$ for the summary rather than single footedness items in ALSPAC, suggesting that in contrast to handedness, multiple items might better capture a genetic component for footedness. One possible interpretation is that multiple items will allow identifying mixed-footed rather than ambipedal individuals, who prefer both feet equally. Similar to Suzuki and Ando [33], our results suggest that the item "foot used to kick a ball", which is often used as the only assessment item, is not the optimal choice to investigate the heritability of footedness. We previously showed that assessing footedness in terms of kicking systematically under-estimates the prevalence of mixed-footedness when compared to assessment using footedness inventories [28]. Overall, there is no one correct measure for laterality items, however, our results demonstrate the importance of reporting data for single items [72] in addition to the aggregates and suggest the value of using multiple items.

All transformed items showed positive correlations on the phenotypic level. Previous research has shown a tendency towards a higher probability of left-sided lateral preferences in left-handers $[28,73]$, suggesting that a common dimension of asymmetry underlies hand, foot, and eye preference [74]. Multivariate SEM analysis supported the presence of one shared genetic factor explaining variance in handedness, footedness, and eyedness, but no unique genetic factors explaining independent variance for individual phenotypes in ALSPAC and the Hong Kong cohort. In ALSPAC, bivariate heritability analysis suggested that up to $37 \%$ of the phenotypic correlation is due to shared genetic effects.

An association between laterality and psychiatric disorders, especially schizophrenia [75], has long been debated. Of the different traits tested, we found suggestive evidence that PRS for IQ were associated with a tendency towards right-handedness, but not with footedness. Similarly, a recent dyslexia GWAS found positive genetic correlation between dyslexia and ambidexterity [76]. A possible explanation for a specific link between cognitive measures and handedness is its association with language. It has been suggested that the higher prevalence of human right- than left-handedness has arisen from a left-hemispheric dominance for manual gestures that gradually incorporated vocalisation [77]. Indeed, right-handers produce more right- than left-handed gestures when speaking [78]. This would suggest that footedness and eyedness are phenotypically secondary to handedness, as has been suggested previously [79].

\section{CONCLUSION}

We assessed the heritability of multiple side preferences using family, genomic, and twin analyses. For footedness, stronger maternal than paternal effects highlight the necessity of examining parent-of-origin effects on the genetic level in future studies. SEM supports a shared genetic factor involved in all three phenotypes without independent genetic factors contributing to footedness and eyedness. The transformed quantitative phenotypes present a heritability that is higher than categorical measures in both molecular and behavioural analyses, suggesting that they might be better suited to identify the underlying genetic factors. 


\section{CODE AVAILABILITY}

Data preparation and visualization were performed using R v.4.0.0. Analysis scripts are available through Github (https://github.com/Judith-Schmitz/heritability_hand_foot_eye)

\section{REFERENCES}

1. Ocklenburg $S$, Hirnstein $M$, Beste $C$, Güntürkün $O$. Lateralization and cognitive systems. Front Psychol. 2014;5:1143.

2. Knecht S, Dräger B, Deppe $M$, Bobe L, Lohmann H, Flöel $A$, et al. Handedness and hemispheric language dominance in healthy humans. Brain. 2000;123:2512-2518.

3. Tzourio-Mazoyer N, Mazoyer B. Variations of planum temporale asymmetries with Heschl's Gyri duplications and association with cognitive abilities: MRI investigation of 428 healthy volunteers. Brain Struct Funct. 2017;222:2711-26.

4. Tzourio-Mazoyer N, Crivello F, Mazoyer B. Is the planum temporale surface area a marker of hemispheric or regional language lateralization? Brain Struct Funct. 2018;223:1217-28.

5. Altarelli I, Leroy F, Monzalvo K, Fluss J, Billard C, Dehaene-Lambertz G, et al. Planum temporale asymmetry in developmental dyslexia: Revisiting an old question. Hum Brain Mapp. 2014;35:5717-35.

6. Elnakib A, Soliman A, Nitzken M, Casanova MF, Gimel'farb G, El-Baz A. Magnetic resonance imaging findings for dyslexia: a review. J Biomed Nanotechnol. 2014;10:2778-805.

7. Oertel-Knöchel V, Knöchel C, Matura S, Prvulovic D, Linden DEJ, van de Ven V. Reduced functional connectivity and asymmetry of the planum temporale in patients with schizophrenia and first-degree relatives. Schizophr Res. 2013;147:331-338.

8. Floris DL, Lai M-C, Auer T, Lombardo MV, Ecker C, Chakrabarti B, et al. Atypically rightward cerebral asymmetry in male adults with autism stratifies individuals with and without language delay. Hum Brain Mapp. 2016;37:230-53.

9. Papadatou-Pastou M, Ntolka E, Schmitz J, Martin M, Munafò MR, Ocklenburg S, et al. Human handedness: a meta-analysis. Psychol Bull. 2020;146:481-524.

10. Schmitz J, Metz GAS, Güntürkün O, Ocklenburg S. Beyond the genome-towards an epigenetic understanding of handedness ontogenesis. Prog Neurobiol. 2017;159:69-89.

11. de Kovel CGF, Carrión-Castillo A, Francks C. A large-scale population study of early life factors influencing left-handedness. Sci Rep. 2019;9:584.

12. Markou P, Ahtam B, Papadatou-Pastou M. Elevated levels of atypical handedness in autism: meta-analyses. Neuropsychol Rev. 2017;27:258-83.

13. Hirnstein $M$, Hugdahl K. Excess of non-right-handedness in schizophrenia: metaanalysis of gender effects and potential biases in handedness assessment. $\mathrm{Br} J$ Psychiatry. 2014;205:260-267.

14. Carter-Saltzman L. Biological and sociocultural effects on handedness: comparison between biological and adoptive families. Science. 1980;209:1263-1265.

15. McManus IC, Bryden MP. The genetics of handedness, cerebral dominance, and lateralization. In: Rapin I, Segalowitz SJ, editors. Handbook of neuropsychology. Elsevier Science. Vol. 6, pp. 115-144 (1992).

16. Pfeifer LS, Schmitz J, Papadatou-Pastou M, Peterburs J, Paracchini S, Ocklenburg S. Handedness in twins: meta-analyses. BMC Psychol. 2022;10:11.

17. Sicotte NL, Woods RP, Mazziotta JC. Handedness in twins: a meta-analysis. Laterality. 1999;4:265-86.

18. Medland SE, Duffy DL, Wright MJ, Geffen GM, Martin NG. Handedness in twins: joint analysis of data from 35 samples. Twin Res Hum Genet. 2006;9:46-53.

19. Medland SE, Duffy DL, Wright MJ, Geffen GM, Hay DA, Levy F, et al. Genetic influences on handedness: data from 25,732 Australian and Dutch twin families. Neuropsychologia. 2009;47:330-337.

20. Coren S, Porac C. Family patterns in four dimensions of lateral preference. Behav Genet. 1980;10:333-48.

21. Reiss M. Genetic associations between lateral signs. Anthropol Anz. 1999;57:61-68.

22. Saudino K, McManus IC. Handedness, footedness, eyedness and earedness in the Colorado Adoption Project. Br J Dev Psychol. 1998;16:167-74.

23. Tran US, Voracek M. Evidence of sex-linked familial transmission of lateral preferences for hand, foot, eye, ear, and overall sidedness in a latent variable analysis. Behav Genet. 2015;45:537-46.

24. Cuellar-Partida G, Tung JY, Eriksson N, Albrecht E, Aliev F, Andreassen OA, et al. Genome-wide association study identifies 48 common genetic variants associated with handedness. Nat Hum Behav. 2021;5:59-70.

25. Oldfield RC. The assessment and analysis of handedness: the Edinburgh inventory. Neuropsychologia. 1971;9:97-113.

26. Sha Z, Schijven D, Carrión-Castillo A, Joliot M, Mazoyer B \& Fisher SE, et al. The genetic architecture of structural left-right asymmetry of the human brain. Nat Hum Behav. https://doi.org/10.1038/s41562-021-01069-w (2021).

27. Kong X-Z, Postema M, Schijven D, Castillo AC, Pepe A \& Crivello F, et al. Largescale phenomic and genomic analysis of brain asymmetrical skew. Cereb Cortex. https://doi.org/10.1093/cercor/bhab075 (2021).
28. Packheiser J, Schmitz J, Berretz G, Carey DP, Paracchini S, Papadatou-Pastou M, et al. Four meta-analyses across 164 studies on atypical footedness prevalence and its relation to handedness. Sci Rep. 2020;10:14501.

29. Dane S, Yildirim S, Ozan E, Aydin N, Oral E, Ustaoglu N, et al. Handedness, eyedness, and hand-eye crossed dominance in patients with schizophrenia: sexrelated lateralisation abnormalities. Laterality. 2009;14:55-65.

30. Giotakos O. Crossed hand-eye dominance in male psychiatric patients. Percept Mot Skills. 2002;95:728-32.

31. Dane S, Balci N. Handedness, eyedness and nasal cycle in children with autism. Int J Dev Neurosci. 2007;25:223-226.

32. Warren DM, Stern M, Duggirala R, Dyer TD, Almasy L. Heritability and linkage analysis of hand, foot, and eye preference in Mexican Americans. Laterality. 2006;11:508-24.

33. Suzuki K, Ando J. Genetic and environmental structure of individual differences in hand, foot, and ear preferences: a twin study. Laterality. 2014;19:113-28.

34. Ooki S. Nongenetic factors associated with human handedness and footedness in Japanese twin children. Environ Health Prev Med. 2006;11:304-12.

35. Boyd A, Golding J, Macleod J, Lawlor DA, Fraser A, Henderson J, et al. Cohort profile: the 'children of the $90 \mathrm{~s}^{\prime}$-the index offspring of the Avon Longitudinal Study of Parents and Children. Int J Epidemiol. 2013;42:111-27.

36. Fraser A, Macdonald-Wallis C, Tilling K, Boyd A, Golding J, Davey Smith G, et al. Cohort profile: the Avon Longitudinal Study of Parents and Children: ALSPAC mothers cohort. Int J Epidemiol. 2013:42:97-110.

37. Wong SWL, Ho CS-H, McBride C, Chow BW-Y, Waye MMY. Less is more in Hong Kong: investigation of biscriptal and trilingual development among Chinese Twins in a (relatively) small city. Twin Res Hum Genet. 2017;20:66-71.

38. Zheng M, McBride $\mathrm{C}, \mathrm{Ho}$ CS-H, Chan JK-C, Choy KW, Paracchini S. Prevalence and heritability of handedness in a Hong Kong Chinese twin and singleton sample. BMC Psychol. 2020;8:37.

39. Brandler WM, Morris AP, Evans DM, Scerri TS, Kemp JP, Timpson NJ, et al Common variants in left/right asymmetry genes and pathways are associated with relative hand skill. PLoS Genet. 2013;9:e1003751.

40. Huisman J. Pedigree reconstruction from SNP data: parentage assignment, sibship clustering and beyond. Mol Ecol Resour. 2017;17:1009-24.

41. Yang J, Lee SH, Goddard ME, Visscher PM. GCTA: a tool for genome-wide complex trait analysis. Am J Hum Genet. 2011;88:76-82.

42. St Pourcain B, Eaves LJ, Ring SM, Fisher SE, Medland S, Evans DM, et al. Developmental changes within the genetic architecture of social communication behavior: a multivariate study of genetic variance in unrelated individuals. Biol Psychiatry. 2018;83:598-606.

43. Papadatou-Pastou M, Martin M, Munafò MR, Jones GV. Sex differences in lefthandedness: a meta-analysis of 144 studies. Psychol Bull. 2008;134:677-99.

44. Buenaventura Castillo C, Lynch AG, Paracchini S. Different laterality indexes are poorly correlated with one another but consistently show the tendency of males and females to be more left- and right-lateralized, respectively. R Soc Open Sci. 2020;7:191700.

45. Benjamini $Y$, Hochberg $Y$. Controlling the false discovery rate: a practical and powerful approach to multiple testing. J R Stat Soc Ser B. 1995;57:289-300.

46. Yang J, Benyamin B, McEvoy BP, Gordon S, Henders AK, Nyholt DR, et al. Common SNPs explain a large proportion of the heritability for human height. Nat Genet. 2010;42:565-569.

47. Fernandez GC, Miller JC. Estimation of heritability by parent-offspring regression. Theor Appl Genet. 1985;70:650-654.

48. Verhoef E, Shapland CY, Fisher SE, Dale PS, St Pourcain B. The developmental origins of genetic factors influencing language and literacy: Associations with early-childhood vocabulary. J Child Psychol Psychiatry. 2020; https://doi.org/ $10.1111 / \mathrm{jcpp} .13327$

49. Boker S, Neale M, Maes H, Wilde M, Spiegel M, Brick T, et al. OpenMx: an open source extended structural equation modeling framework. Psychometrika. 2011;76:306-17.

50. Neale M, Eaves L, Bartels M, Boomsma DI, Posthuma D, Bates T. The 2018 International Workshop on statistical genetic methods for human complex traits. Behav Genet. 2017;47:729-30.

51. Choi SW, O'Reilly PF. PRSice-2: polygenic risk score software for biobank-scale data. Gigascience. 2019;8:giz082.

52. Demontis D, Walters RK, Martin J, Mattheisen M, Als TD, Agerbo E, et al. Discovery of the first genome-wide significant risk loci for attention deficit/hyperactivity disorder. Nat Genet. 2019;51:63-75.

53. Grove J, Ripke S, Als TD, Mattheisen M, Walters RK, Won H, et al. Identification of common genetic risk variants for autism spectrum disorder. Nat Genet. 2019;51:431-44.

54. Mullins N, Forstner AJ, O'Connell KS, Coombes B, Coleman JRI, Qiao Z, et al. Genome-wide association study of more than 40,000 bipolar disorder cases provides new insights into the underlying biology. Nat Genet. 2021;53:817-29.

55. Schizophrenia Working Group of the Psychiatric Genomics Consortium. Biological insights from 108 schizophrenia-associated genetic loci. Nature. 2014:511:421-427. 
56. Savage JE, Jansen PR, Stringer S, Watanabe K, Bryois J, Leeuw CAde, et al. Genome-wide association meta-analysis in 269,867 individuals identifies new genetic and functional links to intelligence. Nat Genet. 2018;50:912-919.

57. Lee JJ, Wedow R, Okbay A, Kong E, Maghzian O, Zacher M, et al. Gene discovery and polygenic prediction from a genome-wide association study of educational attainment in 1.1 million individuals. Nat Genet. 2018;50:1112-21.

58. Francks C, Maegawa S, Laurén J, Abrahams BS, Velayos-Baeza A, Medland SE, et al. LRRTM1 on chromosome 2 p12 is a maternally suppressed gene that is associated paternally with handedness and schizophrenia. Mol Psychiatry. 2007;12:1129-39. 1057

59. Hofmeister RJ, Rubinacci S, Ribeiro DM, Kutalik Z, Buil A, Delaneau O. Parent-oforigin effects in the UK Biobank https://doi.org/10.1101/2021.11.03.467079 (2021).

60. Nudel R, Simpson NH, Baird G, O'Hare A, Conti-Ramsden G, Bolton PF, et al. Genome-wide association analyses of child genotype effects and parent-of-origin effects in specific language impairment. Genes Brain Behav. 2014;13:418-29.

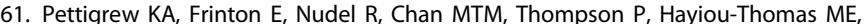
et al. Further evidence for a parent-of-origin effect at the NOP9 locus on language-related phenotypes. J Neurodev Disord. 2016;8:24.

62. Hitchcock TJ, Paracchini S, Gardner A. Genomic imprinting as a window into human language evolution. Bioessays. 2019;41:e1800212.

63. Fennell EB, Satz P, Morris R. The development of handedness and dichotic ear listening asymmetries in relation to school achievement: a longitudinal study. J Exp Child Psychol. 1983;35:248-62.

64. Polyak A, Rosenfeld JA, Girirajan S. An assessment of sex bias in neurodevelopmental disorders. Genome Med. 2015;7:94.

65. de Kovel CGF, Francks C. The molecular genetics of hand preference revisited. Sci Rep. 2019;9:5986.

66. Wiberg A, Ng M, Al Omran Y, Alfaro-Almagro F, McCarthy P, Marchini J, et al. Handedness, language areas and neuropsychiatric diseases: insights from brain imaging and genetics. Brain. 2019;142:2938-47.

67. Paracchini S. Recent advances in handedness genetics. Symmetry. 2021;13:1792.

68. Koboroff A, Kaplan G, Rogers LJ. Hemispheric specialization in Australian magpies (Gymnorhina tibicen) shown as eye preferences during response to a predator. Brain Res Bull. 2008;76:304-306.

69. De Santi A, Bisazza A, Vallortigara G. Complementary left and right eye use during predator inspection and shoal-mate scrutiny in minnows. J Fish Biol. 2002;60:1116-25.

70. Veale JF. Edinburgh Handedness Inventory - Short Form: a revised version based on confirmatory factor analysis. Laterality. 2014;19:164-77.

71. McFarland K, Anderson J. Factor stability of the Edinburgh Handedness Inventory as a function of test-retest performance, age and sex. $\mathrm{Br} J$ Psychol. 1980;71:135-42.

72. Packheiser J, Schmitz J, Pan Y, El Basbasse Y, Friedrich $P$, Güntürkün O, et al. Using mobile EEG to investigate alpha and beta asymmetries during hand and foot use. Front Neurosci. 2020;14:109.

73. Bourassa DC, MCManus IC, Bryden MP. Handedness and eye-dominance: a metaanalysis of their relationship. Laterality. 1996;1:5-34.

74. Porac C. Eye preference patterns among left-handed adults. Laterality. 1997;2:305-16.

75. Crow TJ. The 'big bang' theory of the origin of psychosis and the faculty of language. Schizophr Res. 2008;102:31-52.

76. Doust C, Fontanillas P, Eising E, Gordon SD, Wang Z \& Alagöz G et al. Discovery of 42 genome-wide significant loci associated with dyslexia. medRxiv, https://doi. org/10.1101/2021.08.20.21262334 (2021).

77. Corballis MC. From mouth to hand: gesture, speech, and the evolution of righthandedness. Behav Brain Sci. 2003;26:199-208.

78. Kimura D. Manual activity during speaking - I. Right-handers. Neuropsychologia. 1973;11:45-50.

79. McManus IC, Porac C, Bryden MP, Boucher R. Eye-dominance, writing hand, and throwing hand. Laterality. 1999;4:173-92.

\section{ACKNOWLEDGEMENTS}

We are extremely grateful to all the families who took part in this study, the midwives for their help in recruiting them, and the whole ALSPAC team, which includes interviewers, computer and laboratory technicians, clerical workers, research scientists, volunteers, managers, receptionists and nurses. We also thank Andrew P. Morris and Beate St Pourcain for advising on statistical analyses. The authors would also like to express their gratitude to Sarah Medland, Gabriel Cuellar Partida, and David Evans for providing the handedness GWAS summary statistics. The UK Medical Research Council and Wellcome (Grant ref: 217065/Z/ 19/Z) and the University of Bristol provide core support for ALSPAC. This publication is the work of the authors and SP and JS will serve as guarantors for the analysis of the ALSPAC data presented in this paper. GWAS data were generated by Sample Logistics and Genotyping Facilities at Wellcome Sanger Institute and LabCorp (Laboratory Corporation of America) using support from 23andMe. Support to the genetic analysis was provided by the St Andrews Bioinformatics Unit funded by the Wellcome Trust [grant 105621/Z/14/Z]. The Hong Kong sample was funded through a Collaborative Research Fund from the Hong Kong Special Administrative Region Research Grants Council (CUHK8/CRF/ 13G, and (4054-17WF). JS is funded by the Deutsche Forschungsgemeinschaft (DFG, German Research Foundation, 418445085) and supported by the Wellcome Trust [Institutional Strategic Support fund, Grant number 204821/Z/16/Z]. For the purpose of open access, the author has applied a CC BY public copyright licence to any Author Accepted Manuscript version arising from this submission. SP is funded by the Royal Society (UF150663)

\section{AUTHOR CONTRIBUTIONS}

The study was conceptualised by JS and SP. Administrative efforts and resources were provided by $\mathrm{CM}, \mathrm{CH}$, and $\mathrm{SP} . \mathrm{MZ}, \mathrm{KL}, \mathrm{CM}$, and $\mathrm{CH}$ were involved in data curation and data management. Formal data analysis was performed by JS, MZ, and KL. Funding relevant to the current work was acquired by JS, $\mathrm{CM}, \mathrm{CH}$, and SP. The original draft was written by JS. All authors commented on previous versions of the manuscript, read and approved the final manuscript.

\section{COMPETING INTERESTS}

The authors declare no competing interests.

\section{ADDITIONAL INFORMATION}

Supplementary information The online version contains supplementary material available at https://doi.org/10.1038/s41398-022-01834-z.

Correspondence and requests for materials should be addressed to Silvia Paracchini.

Reprints and permission information is available at http://www.nature.com/ reprints

Publisher's note Springer Nature remains neutral with regard to jurisdictional claims in published maps and institutional affiliations.

(i) Open Access This article is licensed under a Creative Commons Atribution 4.0 International License, which permits use, sharing, adaptation, distribution and reproduction in any medium or format, as long as you give appropriate credit to the original author(s) and the source, provide a link to the Creative Commons license, and indicate if changes were made. The images or other third party material in this article are included in the article's Creative Commons license, unless indicated otherwise in a credit line to the material. If material is not included in the article's Creative Commons license and your intended use is not permitted by statutory regulation or exceeds the permitted use, you will need to obtain permission directly from the copyright holder. To view a copy of this license, visit http://creativecommons. org/licenses/by/4.0/.

(c) The Author(s) 2022 